\title{
Pinning Control Strategy of Multicommunity Structure Networks
}

\author{
Chao Ding, ${ }^{1}$ Hong Yao, ${ }^{2}$ Jun Du, ${ }^{1}$ Xing-zhao Peng, ${ }^{3}$ and Zhe Wang' \\ ${ }^{1}$ Aeronautics and Astronautics Engineering College, Air Force Engineering University, Xian 710038, China \\ ${ }^{2}$ Science College, Air Force Engineering University, Xian 710051, China \\ ${ }^{3}$ Air Force Harbin Flight Academy, Harbin 150009, China \\ Correspondence should be addressed to Chao Ding; mishiding@163.com
}

Received 2 November 2016; Accepted 9 January 2017; Published 1 February 2017

Academic Editor: R. Aguilar-López

Copyright (C) 2017 Chao Ding et al. This is an open access article distributed under the Creative Commons Attribution License, which permits unrestricted use, distribution, and reproduction in any medium, provided the original work is properly cited.

In order to investigate the effects of community structure on synchronization, a pinning control strategy is researched in a class of complex networks with community structure in this paper. A feedback control law is designed based on the network community structure information. The stability condition is given and proved by using Lyapunov stability theory. Our research shows that as to community structure networks, there being a threshold $h_{T} \approx 5$, when coupling strength bellows this threshold, the stronger coupling strength corresponds to higher synchronizability; vice versa, the stronger coupling strength brings lower synchronizability. In addition the synchronizability of overlapping and nonoverlapping community structure networks was simulated and analyzed; while the nodes were controlled randomly and intensively, the results show that intensive control strategy is better than the random one. The network will reach synchronization easily when the node with largest betweenness was controlled. Furthermore, four difference networks' synchronizability, such as Barabási-Albert network, Watts-Strogatz network, Erdös-Rényi network, and community structure network, are simulated; the research shows that the community structure network is more easily synchronized under the same control strength.

\section{Introduction}

The discovery of small-world effect [1] and scale-free characteristics [2] in the real network stirred the enthusiasm for studying complex networks and its content can be divided into two fields, namely, the structure of complex network and the dynamic process in complex networks [3]. The widely existed synchronization is a typical dynamic process. And the synchronization of complex networks means that two or multiple complex networks with the same or similar natures undergo different evolution states through the interaction of systems and gradually approach and eventually reach the identical state [4]. Song of cicadas in the living nature, living clock and making calls, sending emails, the hysteria after a long silence, employees' thoughts synchronization, and the applause in the meeting places in praxeology all belong to the category of synchronization. In the real life, synchronization has immeasurable values [5] in NMR field, signal generator field, laser equipment field, superconducting materials field, confidential communication system, and other fields [5], but the synchronization of periodic routing information in the Internet can lead to the increase of transmission control protocol window and the information congestion of network communication. How to effectively control networks to make the system reach the expected state, namely, "drawing on advantages and avoiding disadvantages," has been a problem scholars try to solve. Pinning control becomes an effective method owing to its simple design, low control costs, and other advantages. Its mechanism is to reach the preset goal of networks by controlling some key nodes. And its core is to solve feasibility and effectiveness problems, namely, whether networks are controllable and at least how many costs will be paid in achieving a controllable overall situation.

Owing to its great practical significance, synchronous control has been widely and deeply studied under the frame of complex networks, which involves the research of parameter identification of network topology [6-8], synchronous control stability theory [9-11], and control methods and 
the selection of control strategies $[12,13]$, and those works mainly focus on the research of scale-free networks and small-world networks. But in reality, the characteristics of community structure of social networks, including the close connection of nodes inside communities and the loose connection of nodes outside communities, are the third most obvious characteristics [14] only behind small-world characteristics and scale-free characteristics, which embodies the thoughts of "birds of a feather flock together," so the research on the control strategies of community structural networks is practically significant. Literature [15-19] studied the synchronization problem of complex networks with community structures relatively early, which mainly studied the influences of network topological parameters on synchronous capacity through phase synchronization method with the conclusion showing that community structure improves the synchronous capacity of networks, but the above researches have not control networks. Literature [20] analyzes the containment and control strategies of networks divided by communities with the results showing that the control of important nodes based on effective information can effectively lower control costs and improve control effects.

In order to figure out the influences of community structure on the synchronous characteristics of networks, the paper studies the pinning control strategies of a kind of multicommunity structure complex networks. It adopts different control strategies of some nodes in different control networks divided by community, designs the control law based on the whole information of networks, applies Lyapunov stability theory to prove the given synchronous conditions of networks, and verifies the correctness of conclusion through numerical simulation. It analyzes the influences of coupling intensity on the synchronous quality of networks and the synchronous characteristics of networks when adopting different control strategies, such as randomly controlling some nodes in networks and deliberately controlling key nodes. Based on that, it analyzes through simulation the synchronous characteristics of networks when the random control and deliberate control strategies of key nodes in the networks with overlapping community structure and compares the synchronous characteristics of the four types of networks.

\section{Description of Model}

2.1. Basic Theories. Considering the complex network consisting of $N$ nodes or $r$ communities, those communities are generally denoted by $C_{1}=\left\{1,2, \ldots, m_{1}\right\}, C_{2}=\left\{m_{1}+1, m_{1}+\right.$ $\left.2, \ldots, m_{1}+m_{2}\right\}$, and $C_{r}=\left\{m_{1}+m_{2}+\cdots+m_{r-1}+1, m_{1}+m_{2}+\right.$ $\left.\cdots+m_{r-1}+2, \ldots, m_{1}+m_{2}+\cdots+m_{r-1}+m_{r}\right\} C_{r}=\left\{m_{1}+m_{2}+\cdots+\right.$ $\left.m_{r-1}+1, m_{1}+m_{2}+\cdots+m_{r-1}+2, \ldots, m_{1}+m_{2}+\cdots+m_{r-1}+m_{r}\right\}$ $\left(1 \leq m_{k} \leq N\right)$, and $\sum_{k=1}^{r} m_{k}=N$ is satisfied. The kinetic equation of its nodes can be described as

$$
\dot{x}_{i}(t)=f_{k}\left(t, x_{i}(t)\right)+c \sum_{p=1}^{r} \sum_{j \in C_{p}} a_{i j} \Gamma x_{j}(t), \quad i \in C_{p} .
$$

In the equation, $x_{i}=\left[x_{i 1}, x_{i 2}, \ldots, x_{i m}\right]^{T}$ represents the state vector of node $i$, and $f_{k}\left(t, x_{i}(t)\right)$ represents the dynamic equation of nodes, generally the continuous nonlinear vector function of a given period. For constant matrix $\Gamma=$ $\operatorname{diag}\left\{\gamma_{1}, \gamma_{2}, \ldots, \gamma_{n}\right\}\left(\gamma_{i}>0\right), \widehat{\Gamma}=\operatorname{diag}\left\{\sqrt{\gamma_{1}}, \sqrt{\gamma_{2}}, \ldots, \sqrt{\gamma_{n}}\right\}$ is the coupling matrix inside the network; constant $c$ is the coupling intensity of node and represents network apology: when there is a connection side between the $i$ th node and the $j$ th node, $a_{i j}=a_{j i}=1$; when there is not, $a_{i j}=a_{j i}=$ 0 defines the diagonal element of coupling matrix as $a_{i i}=$ $-\sum_{j=1, j \neq i}^{N} a_{i j}=-k_{i}$, and $k_{i}$ is the degree of node $i$. In general, matrix $A$ can be written as

$$
A=\left(a_{i j}\right)_{N \times N}=\left[\begin{array}{cccc}
A_{11} & A_{12} & \cdots & A_{1 r} \\
A_{21} & A_{22} & \cdots & A_{2 r} \\
\cdots & \cdots & \cdots & \cdots \\
A_{r 1} & A_{r 1} & \cdots & A_{r r}
\end{array}\right] .
$$

And $A_{u v}=\left(a_{i j}\right)_{i \in C_{u}, j \in C_{v}},(u, v=1,2, \ldots, r)$; the following marks are provided for convenience: $A_{u v}=\left(a_{i j}\right)_{i \in C_{u}, j \in C_{v}}$, $(u, v=1,2, \ldots, r)$; the following marks are provided for convenience: $\sum_{j \in C_{k}} a_{i j}=\alpha_{p k}, i \in C_{k}, \alpha_{k}=\sum_{p=1, p \neq k}^{r} \alpha_{p k}$, $b_{i}^{k}=\sum_{j \in C_{k}} a_{i j}, i \in C_{k}, B_{k}=\operatorname{diag}\left(b_{m_{0}+\cdots+m_{k-1}+1}^{k}, \ldots\right.$, $\left.b_{m_{0}+\cdots+m_{k-1}+m_{k}}^{k}\right)$.

Definition 1. Assume that $s(t) \in R^{n}$ is the given smooth dynamic state. And if any solution of system (1) satisfies

$$
\lim _{t \rightarrow \infty}\left\|x_{i}(t)-s(t)\right\|_{2}=0 \text {, }
$$

it is considered to be completely synchronized to the smooth state $s(t)$.

\section{Assume the following:}

(H1) For any $x, y \in R^{n}$, there is a constant $\theta_{k}$ which satisfies the equation

$$
(x-y)^{T}\left(f_{k}(x)-f_{k}(y)\right) \leq \theta_{k}(x-y)^{T} \Theta(x-y) .
$$

And it is obvious that when the function satisfies Lipschitz continuity, the above assumption is true.

\subsection{Network Topology}

2.2.1. Nonoverlapping Multicommunity Network Model. Through the method proposed in literature [19], the paper produces a network consisting of $N$ nodes or two communities. The two communities, respectively, have $|A|$ and $|B|$ nodes, the probability of interconnection among nodes inside community is $P_{A}=P_{B}=P_{1}$ and the probability of interconnection between community $|A|$ and community $|B|$ is $P_{A B}$. The above method is adopted to produce a nonoverlapping network with two communities with the parameters $|A|=|B|=100, P_{A}=P_{B}=P_{1}=0.5$, and $P_{A B}=0.15$. And the adjacent matrix of networks is shown in Figure 1: the points in the figure mean that the position of corresponding matrix is 1 and the blank in the figure means that the position of corresponding matrix is 0 . 


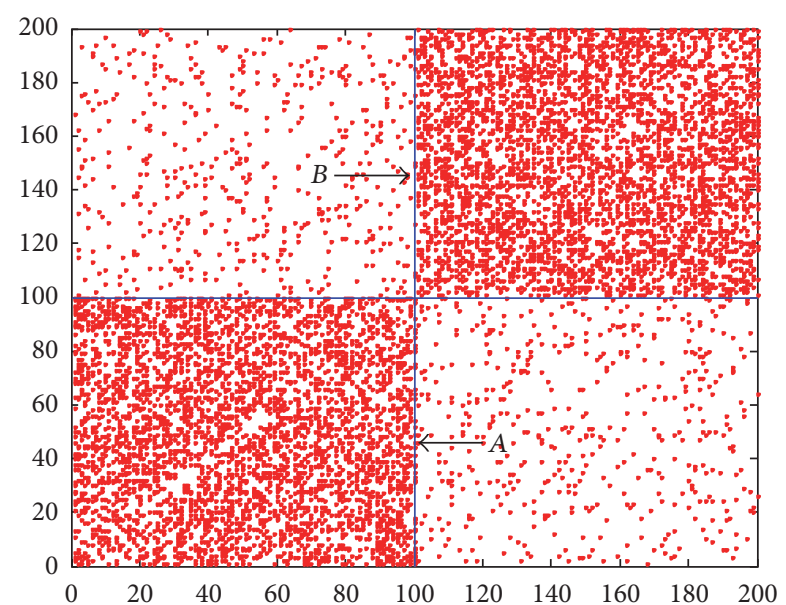

FIGURE 1: The adjacent matrix of nonoverlapping network with two communities.

2.2.2. Overlapping Multicommunity Network Model. The above methods are adopted to produce a network consisting of $N$ nodes or two communities $(A, B)$. The two communities, respectively, have $|A|$ and $|B|$ nodes and include an overlapping community $|O|$ and the nodes in the overlapping network include communities $|A|$ and $|B|$. So the probability of interconnection of nodes inside $N=|A|+|B|+|O|$ is $P_{A}=P_{B}=P_{1}$, the probability of interconnection of nodes inside the overlapping network is $P_{o}$, the probability of interconnection between the nodes of community $|A|$ and community $|B|$ is $P_{A B}$, and the probability of interconnection between the nodes inside communities $|A|$ and $|B|$ and the nodes inside community $|O|$ is $P_{\mathrm{OA}}=P_{\mathrm{OB}}=P_{01}$. The above methods are adopted to produce a network with an overlapping structure with the following parameters $|A|=$ $|B|=100, O=20, P_{A}=P_{B}=0.5, P_{O}=0.6, P_{A B}=$ $0.1, P_{A O}=P_{B O}=0.15$. The adjacent matrix of network is shown in Figure 2: the points in the figure mean that the position of corresponding matrix is 1 and the blank in the figure means that the position of corresponding matrix is 0 .

\section{Pinning Control Strategies of Multicommunity Networks}

Different pinning control strategies are adopted in the networks with different communities to achieve the synchronization of network (1). For the given multicommunity structure network system, assume that the nodes in the first $l(1 \leq l \leq r-1)$ communities are controlled, the control law is designed based on the community structure information of networks, and the control input of system (1) is

$$
u_{i}= \begin{cases}\sum_{j \in C_{k}} a_{i j}\left(x_{j}(t)-s(t)\right), & i \in C_{k}, k=1,2, \ldots, l \\ 0, & \text { other. }\end{cases}
$$

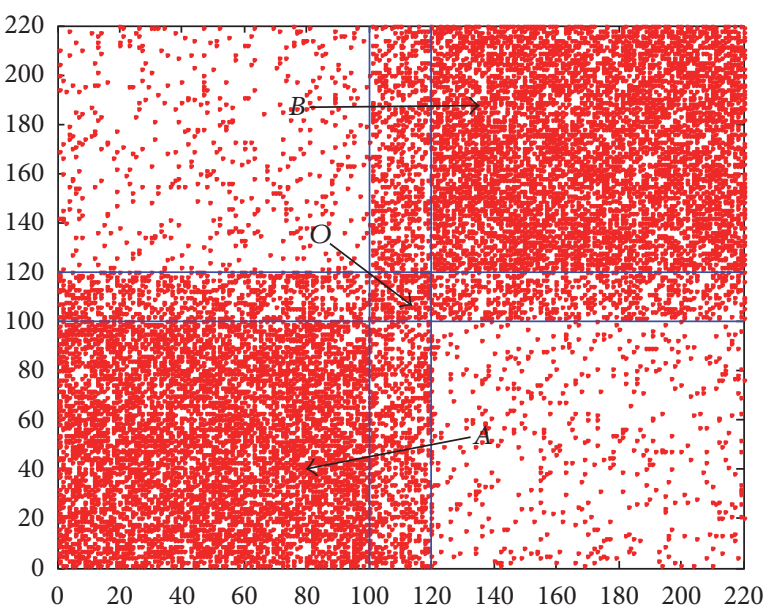

FIGURE 2: The adjacent matrix of overlapping network with two communities.

The control input fully considers network information. The controlled system model is

$$
\begin{gathered}
\dot{x}_{i}(t)=f_{k}\left(x_{i}(t)\right)+c \sum_{p=1}^{r} \sum_{j \in C_{p}} a_{i j} \Gamma x_{j}(t) \\
-\sum_{j \in C_{k}} a_{i j}\left(x_{j}(t)-s(t)\right), \\
i \in C_{k}, k=1,2, \ldots, r \\
\dot{x}_{i}(t)=f_{k}(x)+c \sum_{p=1}^{r} \sum_{j \in C_{p}} a_{i j} \Gamma x_{j}(t), \\
i \in C_{k}, k=l+1, \ldots, r .
\end{gathered}
$$

Theorem 2. Under the precondition of (H1), if system (1) satisfies

$$
\begin{aligned}
\alpha_{k} I_{m_{k}}-B_{k} I_{m_{k}}+\theta_{k} I_{m_{k}}< & 0, \\
& k=1,2, \ldots, l \\
+\theta_{k} I_{m_{k}}+A_{k k}^{T}+A_{k k}< & 0, \\
k & =l+1, \ldots, r,
\end{aligned}
$$$$
\alpha_{k} I_{m_{k}}-B_{k} I_{m_{k}}+\theta_{k} I_{m_{k}}+A_{k k}^{T}+A_{k k}<0,
$$

then network (1) can be synchronized under control (5). 
Demonstration. Error variable is defined as $e_{i k}=x_{i}(t)-$ $s(t), i \in C_{k}, k=1, \ldots, r$, and the corresponding synchronous variance equation of system (6) is

$$
\begin{aligned}
& \dot{e}_{i k}(t)=\bar{f}_{k}\left(e_{i k}\right)+c \sum_{p=1}^{r} \sum_{j \in C_{p}} a_{i j} \Gamma e_{j p}(t)-\sum_{j \in C_{k}} a_{i j} e_{j k}, \\
& \quad i \in C_{k}, k=1, \ldots, l \\
& \dot{e}_{i k}(t)=\bar{f}_{k}\left(e_{i k}\right)+c \sum_{p=1}^{r} \sum_{j \in C_{p}} a_{i j} \Gamma e_{j p}(t),
\end{aligned}
$$$$
i \in C_{k}, k=l+1, \ldots, r .
$$

And $\bar{f}_{k}\left(e_{i k}\right)=f_{k}\left(t, x_{i}(t)\right)-f_{k}(t, s(t))$. For convenience, Lyapunov function is defined as

$$
V(t)=\frac{1}{2} \sum_{k=1}^{r} e_{k}^{T}(t) e_{k}(t)=\frac{1}{2} \sum_{k=1}^{r} \sum_{i \in C_{k}} e_{i k}^{T}(t) e_{i k}(t) .
$$

The calculation of derivative of $V(t)$ is

$$
\begin{aligned}
& \frac{d V}{d t}=\sum_{k=1}^{r} \sum_{i \in C_{k}} e_{i k}^{T}(t) \\
& \cdot\left[f_{k}\left(t, x_{i}(t)\right)-f_{k}(t, s(t))+c \sum_{p=1}^{r} \sum_{j \in C_{p}} a_{i j} \Gamma e_{j p}(t)\right] \\
& -c \sum_{k=1}^{l} \sum_{j \in C_{k}} \sum_{i}^{N} a_{i j} e_{j k}^{T}(t) \Gamma e_{j k}(t) \leq \sum_{k=1}^{r} \sum_{i \in C_{k}} \theta_{k} e_{i k}^{T}(t) \\
& \cdot \Gamma e_{j k}(t)+c \sum_{k=1}^{r} \sum_{i \in C_{k}} \sum_{p=1}^{r} \sum_{j \in C_{p}} a_{i j} e_{i k}^{T}(t) \Gamma e_{j p}(t) \\
& -c \sum_{k=1}^{l} e_{k}^{T}(t)\left(\frac{A_{k k}{ }^{T}+A_{k k}}{2} \otimes \Gamma\right) e_{k}(t), \\
& c \sum_{k=1}^{r} \sum_{i \in C_{k}} \sum_{p=1}^{r} \sum_{j \in C_{p}} a_{i j} e_{i k}^{T}(t) \Gamma e_{j p}(t) \\
& \quad=c \sum_{k=1}^{r} \sum_{i \in C_{k}} \sum_{j \in C_{k}} a_{i j} e_{i k}^{T}(t) \Gamma e_{j k}(t) \\
& \quad+c \sum_{k=1}^{r} \sum_{i \in C_{k}} \sum_{p=1, p \neq k}^{r} \sum_{j \in C_{p}} a_{i j}\left(\widehat{\Gamma} e_{i k}(t)\right)^{T}\left(\widehat{\Gamma} e_{j p}(t)\right) \\
& \quad \leq c \sum_{k=1}^{r} e_{k}^{T}(t)\left(\frac{A_{k k}{ }^{T}+A_{k k}}{2} \otimes \Gamma\right) e_{k}(t)+\frac{c}{2} \\
&
\end{aligned}
$$

$$
\begin{aligned}
& \cdot \sum_{k=1}^{r} \sum_{i \in C_{k}} \sum_{p=1, p \neq k}^{r} \sum_{j \in C_{p}} a_{i j} \\
& \cdot\left(e_{i k}^{T}(t) \Gamma e_{i k}(t)+e_{j p}^{T}(t) \Gamma e_{j p}(t)\right)=c \sum_{k=1}^{r} e_{k}^{T}(t) \\
& \cdot\left(\frac{A_{k k}^{T}+A_{k k}}{2} \otimes \Gamma\right) e_{k}(t)+\frac{c}{2}
\end{aligned}
$$$$
\cdot \sum_{k=1}^{r} \sum_{i \in C_{k}} \sum_{p=1, p \neq k} \alpha_{p k} e_{i k}^{T}(t) \Gamma e_{j k}(t)+\frac{c}{2}
$$$$
\cdot \sum_{p=1}^{r} \sum_{j \in C_{p}}\left(\sum_{k=1}^{r} \sum_{i \in C_{k}} a_{i j}\right) e_{j p}^{T}(t) \Gamma e_{j p}(t)-\frac{c}{2}
$$$$
\cdot \sum_{k=1}^{r} \sum_{i \in C_{k}}\left(\sum_{j \in C_{k}} a_{i j}\right) e_{j k}^{T}(t) \Gamma e_{j k}(t)=\frac{c}{2} \sum_{k=1}^{r} e_{k}^{T}(t)
$$$$
\cdot\left(\alpha_{k} I_{m_{k}} \otimes \Gamma\right) e_{k}(t)-\frac{c}{2} \sum_{k=1}^{r} e_{k}^{T}(t)\left(B_{k} I_{m_{k}} \otimes \Gamma\right) e_{k}(t)
$$$$
+c \sum_{k=1}^{r} e_{k}^{T}(t)\left(\frac{A_{k k}^{T}+A_{k k}}{2} \otimes \Gamma\right) e_{k}(t) .
$$

The following equation is produced by combining (10) and (11):

$$
\begin{aligned}
& \frac{d V}{d t}=\frac{c}{2} \sum_{k=1}^{r} e_{k}^{T}(t)\left(\theta_{k} I_{m_{k}} \otimes \Gamma\right) e_{k}(t)-c \sum_{k=1}^{l} e_{k}^{T}(t) \\
& \cdot\left(\frac{A_{k k}{ }^{T}+A_{k k}}{2} \otimes \Gamma\right) e_{k}(t)+\frac{c}{2} \sum_{k=1}^{r} e_{k}^{T}(t) \\
& \cdot\left(\alpha_{k} I_{m_{k}}-B_{k} I_{m_{k}}+A_{k k}{ }^{T}+A_{k k}\right) \otimes \Gamma e_{k}(t)=\frac{c}{2} \\
& \cdot \sum_{k=1}^{l} e_{k}^{T}(t)\left(\alpha_{k} I_{m_{k}}-B_{k} I_{m_{k}}+\theta_{k} I_{m_{k}}\right) \otimes \Gamma e_{k}(t)+\frac{c}{2} \\
& \cdot \sum_{k=l+1}^{r} e_{k}^{T}(t)\left(\alpha_{k} I_{m_{k}}-B_{k} I_{m_{k}}+\theta_{k} I_{m_{k}}+A_{k k}{ }^{T}+A_{k k}\right) \\
& \otimes \Gamma e_{k}(t)<0 .
\end{aligned}
$$

According to Lyapunov stability theory,

$$
V(t)=\lim _{t \rightarrow+\infty} \sum_{k=1}^{r} \sum_{i \in C_{k}} e_{i k}^{T}(t) e_{i k}(t)=0 .
$$

The controlled network is synchronous. The demonstration is over. 


\section{Numerical Simulation and Analysis}

The previous section provides the demonstration of the design and stability of pinning control law of multicommunity structure complex networks. This section first verifies the feasibility of the pinning control of multicommunity structure network; then, it studies the effectiveness of pinning control, especially the synchronous performance of some nodes in randomly controlled networks and the key nodes in the deliberately controlled networks. For deliberate control, the synchronous characteristics when the nodes with the largest betweenness and the highest degree are controlled in different manners subject to communities are studied mainly. The section also studies the pinning control strategies of networks with overlapping community structure in the further analysis, especially the synchronous characteristics of networks when the key nodes of overlapping part are controlled, and compares and chooses the quality of different control strategies of key nodes through simulation results.

4.1. Pinning Control Effective. This section verifies the correctness and feasibility through an example and considers the complex network system consisting of two communities and 20 nodes in (14) with the description of specific model as follows:

$$
\begin{aligned}
\dot{x}_{i}(t)= & 2 x_{i}(t)+8 \tanh \left(x_{i}(t)\right)+c \sum_{p=1}^{r} \sum_{j \in C_{p}} a_{i j} \Gamma x_{j}(t) \\
& -\sum_{j \in C_{k}} a_{i j}\left(x_{j}(t)-s(t)\right), \quad i=1,11 \\
\dot{x}_{i}(t)= & 2 x_{i}(t)+8 \tanh \left(x_{i}(t)\right) \\
& +c \sum_{p=1}^{r} \sum_{j \in C_{p}} a_{i j} \Gamma x_{j}(t), \quad \text { other. }
\end{aligned}
$$

Assume that $c=5, \Gamma=I$, the adjacent matrix of networks is shown in Figure 1. We add one controller between the first node (belonging to the first community) and the eleventh node (belonging to the second community). And the synchronous effects of networks under the control law raised in the paper are shown in Figure 3.

We can see from Figure 3 that when the first node and the eleventh node are controlled the whole network system reaches complete synchronization after 1.4 seconds, which proves the feasibility of the control strategy raised in the paper and the feasibility of achieving the complete synchronization of system by controlling the nodes of community.

4.2. The Effectiveness Analysis of Pinning Control. The section studies the effectiveness problem of pinning control and the influences of various nodes in networks on the pinning control effects. The synchronous quality of containment and control described by definition error function $E(t)$ is as follows:

$$
E(t)=\sqrt{\sum_{i=1}^{N} \frac{\left|\dot{x}_{i}(t)-s(t)\right|}{N}} .
$$

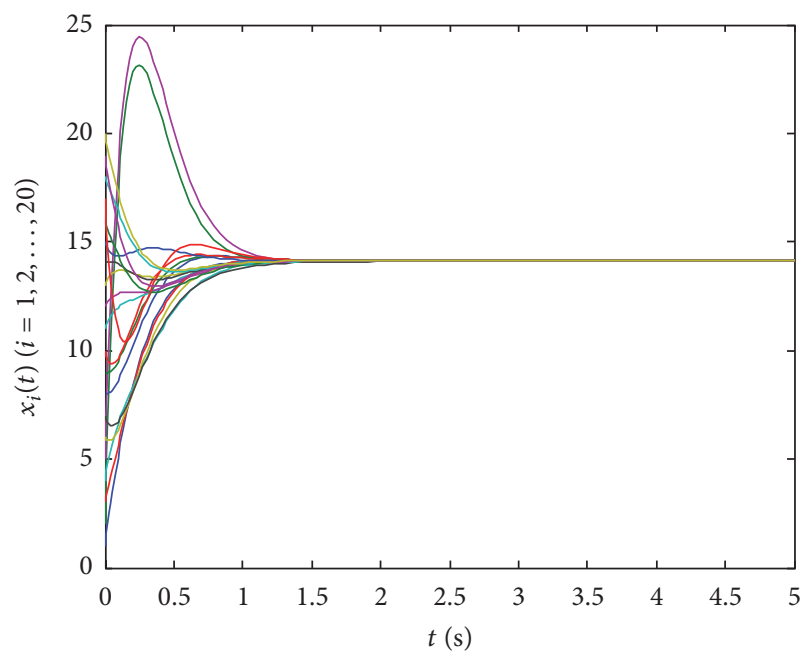

FIGURE 3: The evolution diagram of state of nodes with time.

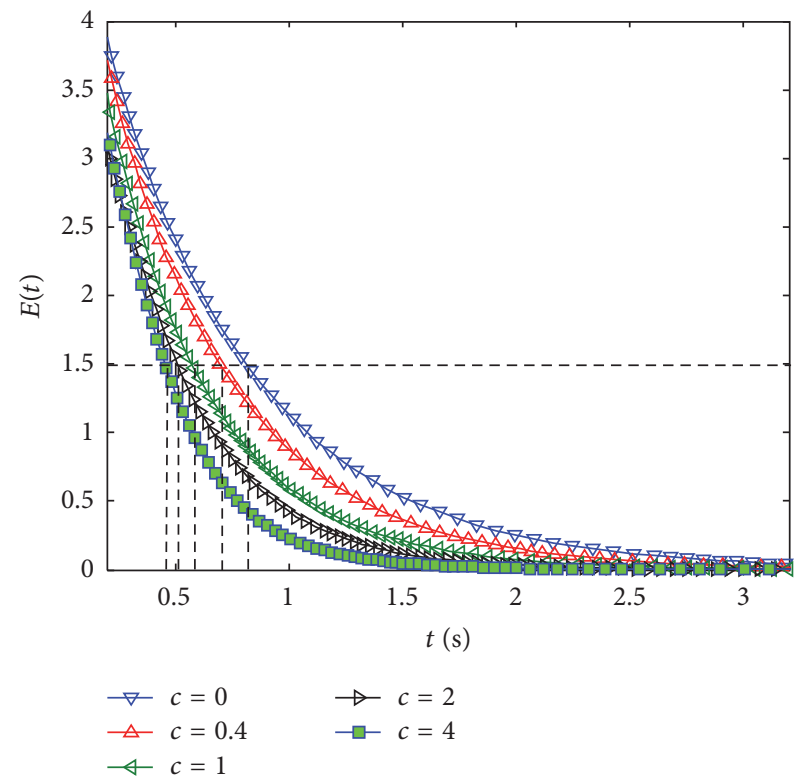

Figure 4: The curve of $E(t) \sim t$ when $c$ is set as $0,0.4,1,2$, and 4 .

4.2.1. The Influences of Coupling Intensity on Synchronous Characteristics. For the influences of coupling intensity $c$ of networks on the synchronous pinning control effects, the system model is marked with (14), the adjacent matrix of networks is shown in Figure 1, the change curve of $E(t)$ with time when the nodes with the highest degree in the networks are controlled, and coupling intensity $c$ is, respectively, 6 , 8,10 , and 12 as shown in Figure 5. In order to reduce the contingency, the results in Figures 4 and 5 adopt the average value of 100 times of simulation.

As shown in Figure 4, when $c<4$ and after $t, E(t)$ gradually decreases as $c$ increases; namely, when $c<4$, the synchronous quality of networks improves with the increase of coupling intensity; as shown in Figure 5, when $c>6$, the synchronous quality of networks decreases with the strengthening of coupling intensity. So, we assume that 


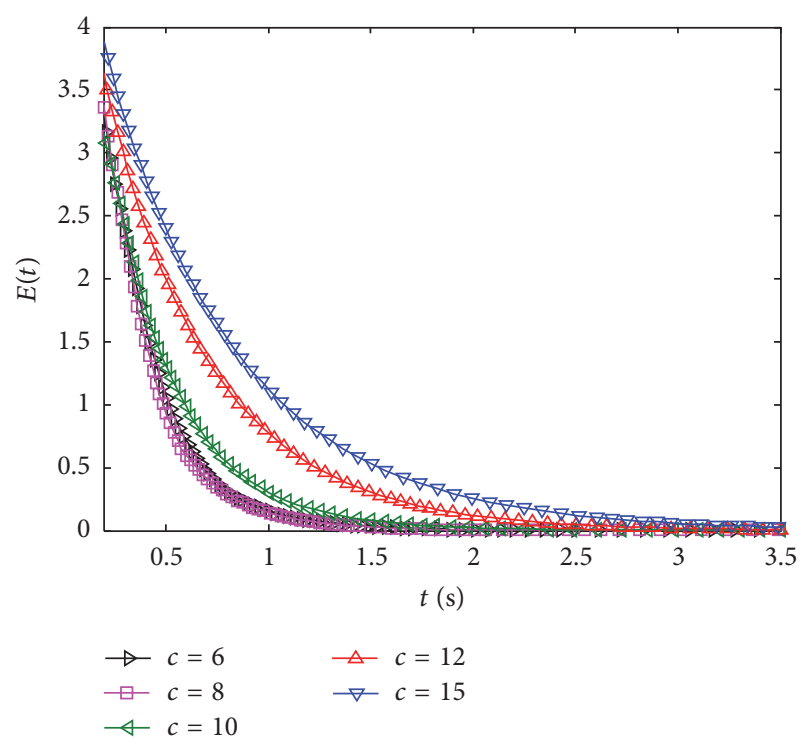

Figure 5: The curve of $E(t) \sim t$ when $c$ is set as 6, 8, 10, 12, and 15 .

there is threshold value $c_{T}$ in $4<c<6$; when $c>$ $c_{T}$, the synchronous quality of networks is proportional to coupling intensity; and when $c>c_{T}$, the synchronous quality of networks is inversely proportional to coupling intensity. In order to verify the assumption, we set a variable $\alpha$ representing the time $t$ after which the error value of networks $E(t)$ is lower than $\alpha$. Taking $\alpha=1.5$ as an example in Figure 4, when $c=2$, the abscissa of the intersection point between curve $E \sim t$ and straight line $E=1.5$ is about 0.5 meaning that when $t<t_{1.5}=0.5$, the synchronous error of networks is lower than $\alpha=1.5$; when $t>t_{1.5}=0.5$, the synchronous error of networks is higher than $\alpha=1.5$. It is clear that the larger $t_{\alpha}$ is, the longer time networks need to take to control the synchronous errors in the corresponding range, so the poorer the synchronous capacity of networks is. Figure 6 is the corresponding $t_{\alpha} \sim c$ curve of different $\alpha$.

As shown in Figure 6, there is indeed a threshold value $c_{T}$; when $c \leq c_{T}$, the larger $c$ is, the smaller $t_{\alpha}$ is, so the stronger the synchronous capacity of networks is; when $c \geq c_{T}$, the larger $c$ is, the larger $t_{\alpha}$ is, so the weaker the synchronous capacity of networks is. As shown in Figure 6, the threshold value is about 5 ; namely, $h_{T} \approx 5$. Therefore, the above simulation results show that when the networks with community structure are contained and controlled, the relation between the coupling intensity and synchronous characteristics of networks is not monotonically increasing or decreasing but has a threshold value, and the threshold value is greatly practically significant for effectively controlling networks.

4.2.2. Pinning Control Strategies of Nonoverlapping Multicommunity Networks. Aiming at the nonoverlapping multicommunity structure model, simulate the synchronous quality of networks in the random control of the nodes of networks and the deliberate control of the nodes with the maximum (minimum) betweenness of their community and the nodes

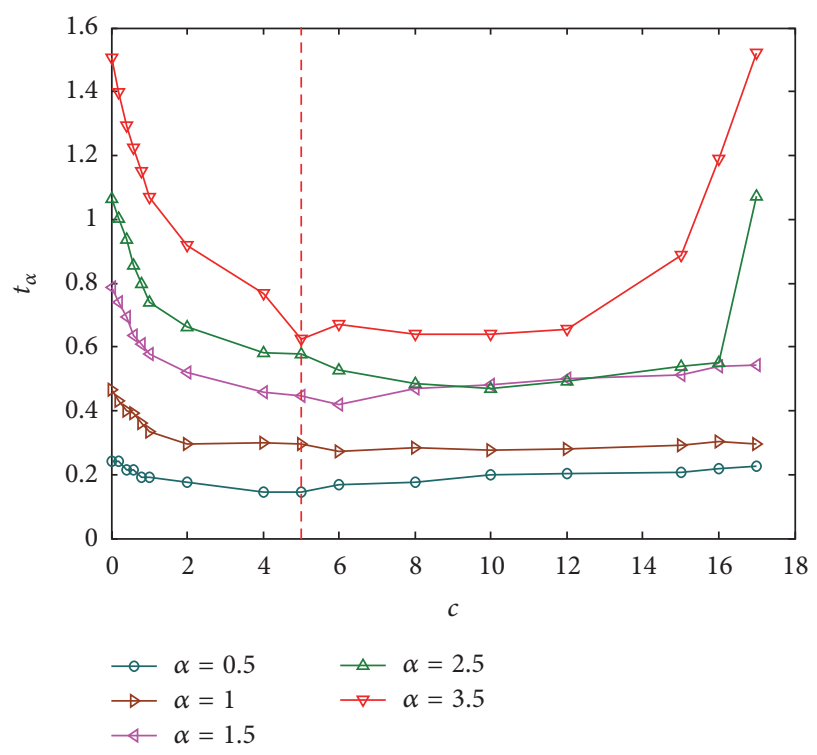

FIGURE 6: The corresponding $t_{\alpha} \sim c$ curve of different $\alpha$.

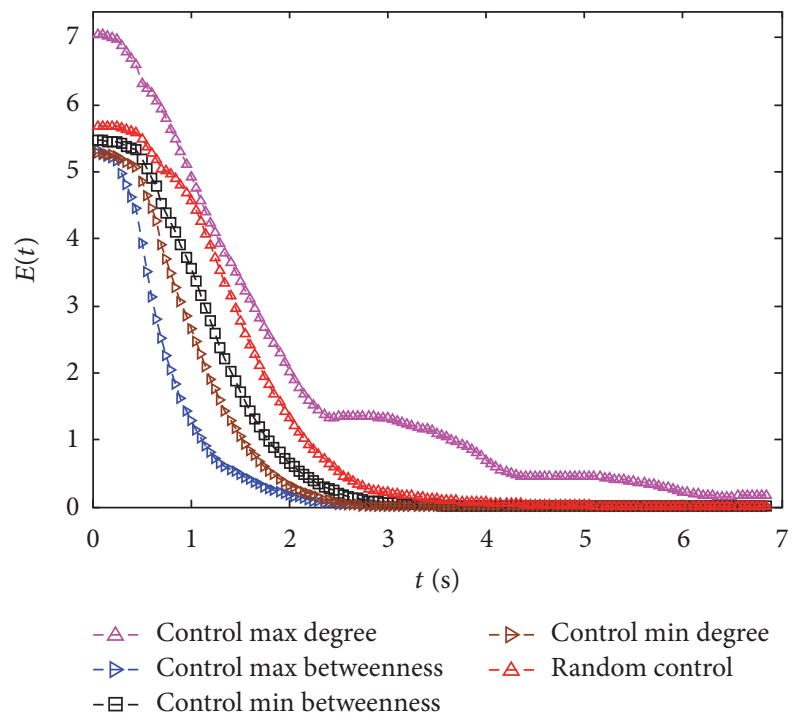

FIGURE 7: $E(t) \sim t$ curve of nonoverlapping networks under different control strategies.

with the maximum (minimum) degree of their community. The system model (14) is adopted, the parameters of networks are $c=5, \Gamma=I$, and the adjacent matrix of networks is shown in Figure 1. In order to reduce accidental results, the average value of 100 times of simulation is adopted. And the change curve of $E(t)$ with time under different control strategies is shown in Figure 7.

As shown in Figure 7, when the same amount of nodes is controlled, deliberate control has better synchronous quality than random control; when the nodes with the maximum degree, the nodes with the maximum betweenness, and the nodes with the minimum betweenness of different communities are deliberately controlled, the ranking of synchronous 


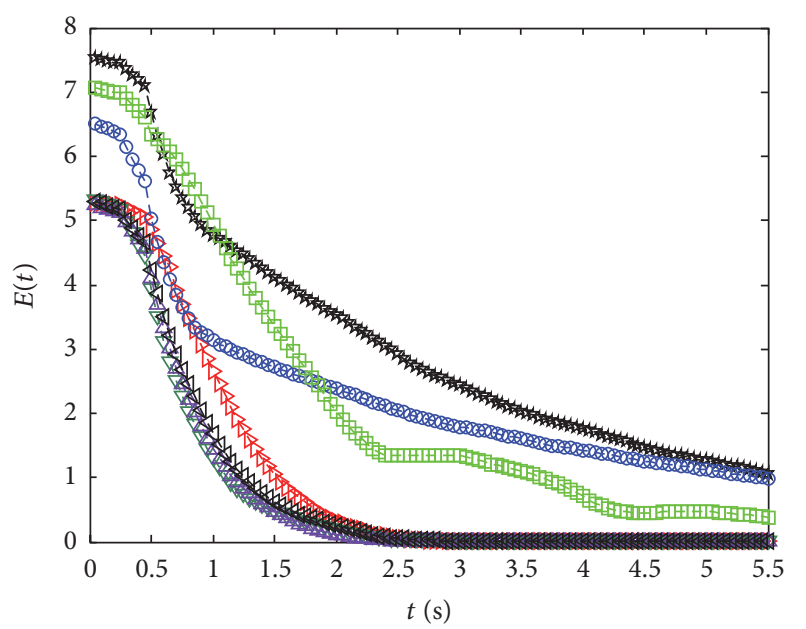

$-\nabla-$ Control min overlap betweenness

$-\nabla-$ Control max overlap betweenness

-A- Control max overlap degree

一古- Random control

$-\varangle$ - Control max nonoverlap degree

$-\ominus-$ Control min nonoverlap degree

$-\square-$ Control min overlap degree

FIGURE 8: $E(t) \sim t$ curve of overlapping networks under different control strategies.

quality of system is the nodes with the maximum betweenness, the nodes with the maximum degree, the nodes with the minimum degree, and the nodes with the minimum degree. Under the same coupling intensity and control costs, it is easier for the control of the nodes with the maximum betweenness of networks to achieve the synchronization of networks. The nodes with the maximum betweenness in the networks are generally "opinion leader" and have a relatively large border, which can furthest influence the synchronization of its surrounding nodes after being controlled, which is in line with our common sense of life.

4.2.3. Pinning Control Strategies of Overlapping Multicommunity Networks. Aiming at the overlapping multicommunity structure model, simulate the synchronous quality of networks in the random control of the nodes of networks and the deliberate control of the nodes with the maximum (minimum) betweenness of overlapping part and the nodes with the maximum (minimum) betweenness of overlapping part and the maximum (minimum) degree of overlapping part. The system model (14) is adopted, the parameters of networks are $c=5, \Gamma=I$, and the adjacent matrix of networks is shown in Figure 2. In order to reduce accidental results, the average value of 100 times of simulation is adopted. And the change curve of $E(t)$ with time under different control strategies is shown in Figure 8.

As shown in Figure 8, under the same control strength, the synchronous quality of deliberate control is better than that of random control. For deliberate control, when the nodes with the maximum betweenness of overlapping part are controlled, it has the best synchronous effects, and when

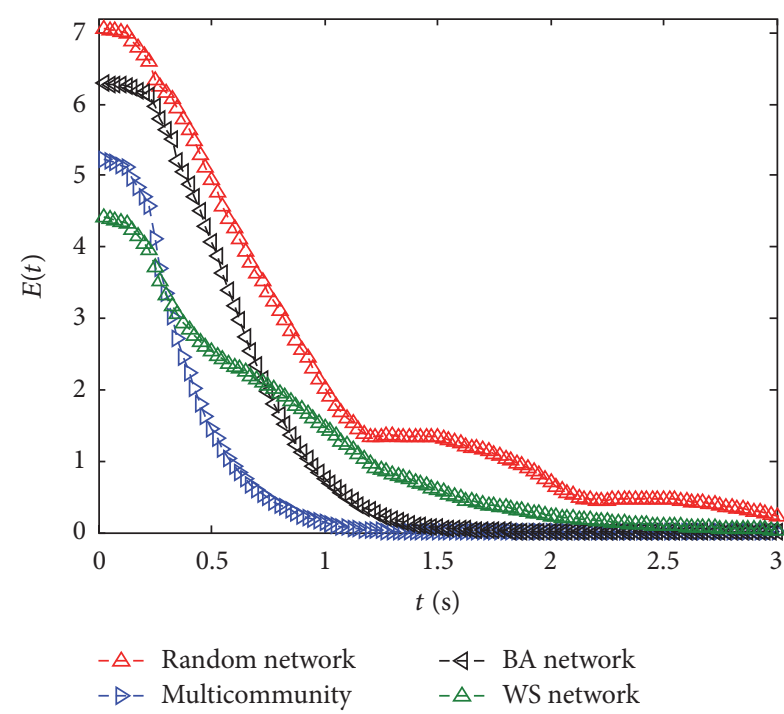

FIGURE 9: $E(t) \sim t$ curve of different networks under the same control strategies.

the nodes with the minimum betweenness of overlapping part are controlled, it has the poorest synchronous effects. The nodes of overlapping part, as the bridge between different communities, can more effectively expand the control to its surrounding nodes, so the deliberate control of the nodes with the maximum betweenness of overlapping part can achieve the best control effects at the same control costs.

4.2.4. The Control Effects of Different Control Networks. In order to further study the control effects of different network models, the synchronous quality of the deliberate control of the nodes with the maximum betweenness of scale-free networks (BA), small-world networks (WS), random networks (ER), and multicommunity structure networks under the same control strength is analyzed through simulation. In order to reduce the intervention of irrelevant factors in simulation, the different network models should have the same average degree. System model (14) is used, the parameters of networks are $c=5, \Gamma=I$, and the average degree of model is 4 . In order to reduce accidental errors, the average value of 1000 times of simulation results is adopted. The control effects of different networks under the functioning of deliberate control are shown in Figure 9.

As shown in Figure 9, when the nodes with the maximum betweenness are controlled deliberately, multicommunity structure networks have the best synchronous quality and random networks have the poorest synchronous quality. It is found in the analysis of the betweenness distribution of the four network nodes made in order to look for the cause of the phenomenon that when the uniformity is the same, multicommunity networks have the least uniform distribution of betweenness and random networks have the most uniform distribution of random. The trend is the same as its synchronous characteristics, so it can be concluded that the distribution uniformity of betweenness of networks 
is inversely proportional to the synchronous effects of key nodes control.

\section{Conclusions}

This paper studies the pinning control problem of a kind of multicommunity complex networks, designs the pinning control law based on the community structure information of networks, which not only effectively takes advantage of network topology but also avoids excessive calculation, applies Lyapunov stability theory to prove the given synchronization conditions of networks, solves the feasibility problem of pinning control, and verifies the correctness of theories through numerical simulation. While analyzing the effectiveness of pinning control, it also analyzes the influences of coupling intensity on the synchronous quality of networks, with the conclusion showing when the synchronous quality of networks is positively proportional to coupling intensity and when the synchronous quality of networks is inversely proportional to coupling intensity. It, aiming at the simulation of nonoverlapping community networks, analyzes the key nodes in randomly controlled networks and deliberately controlled networks with the results showing that deliberate control can achieve relatively good synchronous control effects and the control of the nodes with the maximum betweenness can achieve the best synchronous control effects; it, aiming at the simulation of overlapping structural networks, analyzes the synchronous characteristics of randomly controlled and deliberately controlled key nodes networks with the results showing that the control of the nodes with the maximum betweenness at the overlapping part can achieve the best synchronous quality. And it further analyzes the synchronous characteristics of BA networks, WS small-world networks, ER networks, and multicommunity networks with the results showing that it is easier for multicommunity networks to achieve synchronization under the same control intensity.

\section{Competing Interests}

The authors declare that they have no competing interests.

\section{Acknowledgments}

This project is supported by the Shaanxi Science Foundation of China (Grant no. 2012JM8035).

\section{References}

[1] D. J. Watts and S. H. Strogatz, "Collective dynamics of 'smallworld' networks," Nature, vol. 393, no. 6684, pp. 440-442, 1998.

[2] A.-L. Barabási and R. Albert, "Emergence of scaling in random networks," Science, vol. 286, no. 5439, pp. 509-512, 1999.

[3] G. Liu and Y.-S. Li, "Study on the congestion phenomena in complex network based on gravity constraint," Acta Physica Sinica, vol. 61, no. 10, Article ID 108901, 2012.

[4] J.-L. Liu, "Research on synchronization of complex networks with random nodes," Acta Physica Sinica, vol. 62, no. 4, Article ID 040503, 2013.
[5] I. I. Blekhman, Synchronization in Science and Technology, American Society of Mechanical Engineers, 1988.

[6] D. Xu, Y. Li, and T.-J. Wu, "Improving consensus and synchronizability of networks of coupled systems via adding links," Physica A: Statistical Mechanics and Its Applications, vol. 382, no. 2, pp. 722-730, 2007.

[7] J. Zhou, J.-A. Lu, and J. Lü, "Pinning adaptive synchronization of a general complex dynamical network," Automatica, vol. 44, no. 4, pp. 996-1003, 2008.

[8] H. Liu, J.-A. Lu, J. Lü, and D. J. Hill, "Structure identification of uncertain general complex dynamical networks with time delay," Automatica, vol. 45, no. 8, pp. 1799-1807, 2009.

[9] C. Huang, D. W. Ho, and J. Lu, "Synchronization analysis of a complex network family," Nonlinear Analysis: Real World Applications, vol. 11, no. 3, pp. 1933-1945, 2010.

[10] X. Guo and J. Li, "A new synchronization algorithm for delayed complex dynamical networks via adaptive control approach," Communications in Nonlinear Science and Numerical Simulation, vol. 17, no. 11, pp. 4395-4403, 2012.

[11] T. H. Lee, J. H. Park, D. H. Ji, O. M. Kwon, and S. M. Lee, "Guaranteed cost synchronization of a complex dynamical network via dynamic feedback control," Applied Mathematics and Computation, vol. 218, no. 11, pp. 6469-6481, 2012.

[12] Y. Liang, X. Wang, and J. Eustace, "Adaptive synchronization in complex networks with non-delay and variable delay couplings via pinning control," Neurocomputing, vol. 123, pp. 292-298, 2014.

[13] L. Wang and Y.-X. Sun, "Robustness of pinning a general complex dynamical network," Physics Letters, Section A: General, Atomic and Solid State Physics, vol. 374, no. 15-16, pp. 1699-1703, 2010.

[14] M. E. J. Newman and M. Girvan, "Finding and evaluating community structure in networks," Physical Review E, vol. 69, no. 2, Article ID 026113, 2004.

[15] J. A. Almendral, I. Leyva, D. Li, I. Sendiña-Nadal, S. Havlin, and S. Boccaletti, "Dynamics of overlapping structures in modular networks," Physical Review E, vol. 82, no. 1, Article ID 016115, 2010.

[16] X.-H. Wang, L.-C. Jiao, and J.-S. Wu, "Phase synchronization on small-world networks with community structure," Chinese Physics B, vol. 19, no. 2, Article ID 020501, 2010.

[17] T. Zhou, M. Zhao, G. Chen, G. Yan, and B.-H. Wang, "Phase synchronization on scale-free networks with community structure," Physics Letters A, vol. 368, no. 6, pp. 431-434, 2007.

[18] D. Li, I. Leyva, J. A. Almendral et al., "Synchronization interfaces and overlapping communities in complex networks," Physical Review Letters, vol. 101, no. 16, Article ID 168701, 2008.

[19] J. Wu, X. Wang, and L. Jiao, "Synchronization on overlapping community network," Physica A: Statistical Mechanics and its Applications, vol. 391, no. 3, pp. 508-514, 2012.

[20] Y.-Y. Wu, W. Wei, and G.-Y. Li, "Pinning control of complex networks via network division," Journal of Zhejiang University (Engineering Science), vol. 45, no. 3, pp. 495-502, 2011. 


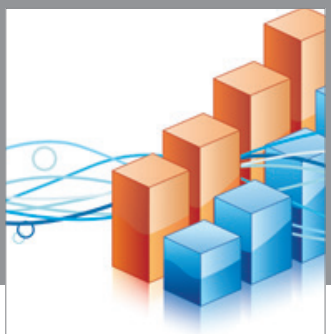

Advances in

Operations Research

vatem alat4

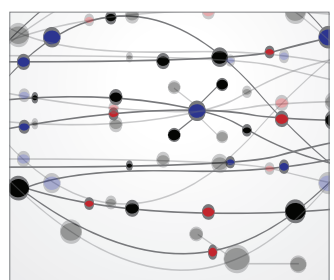

\section{The Scientific} World Journal
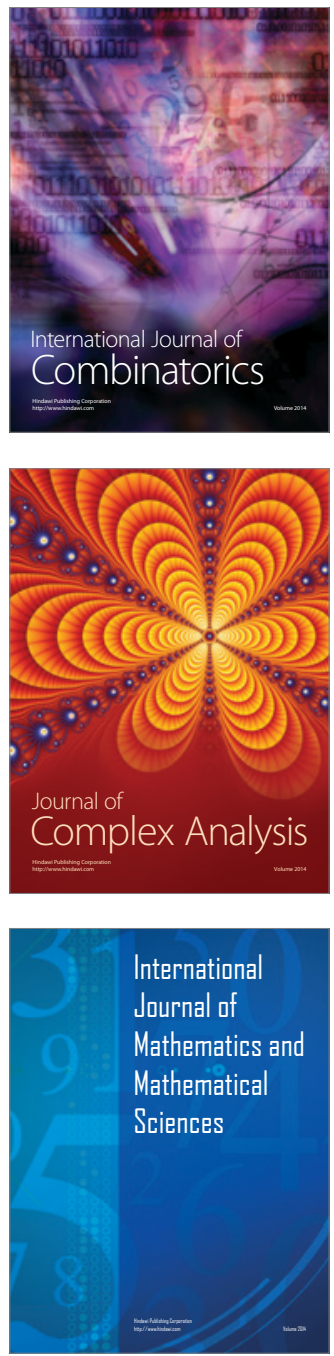
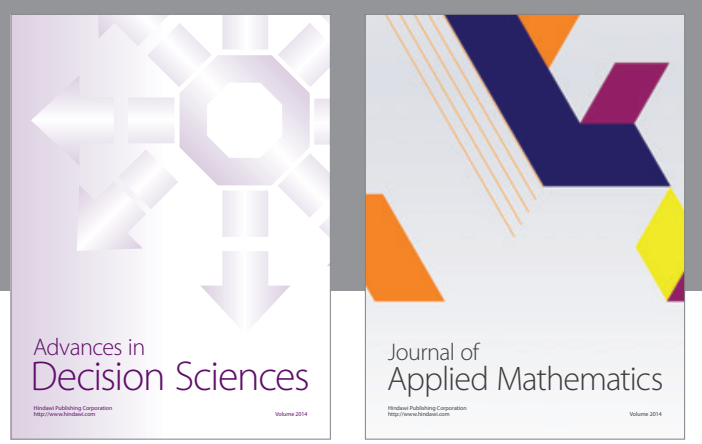

Algebra

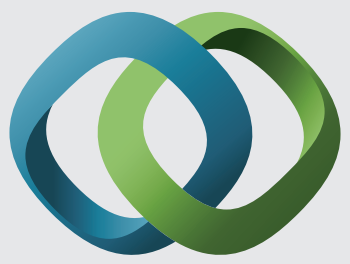

\section{Hindawi}

Submit your manuscripts at

https://www.hindawi.com
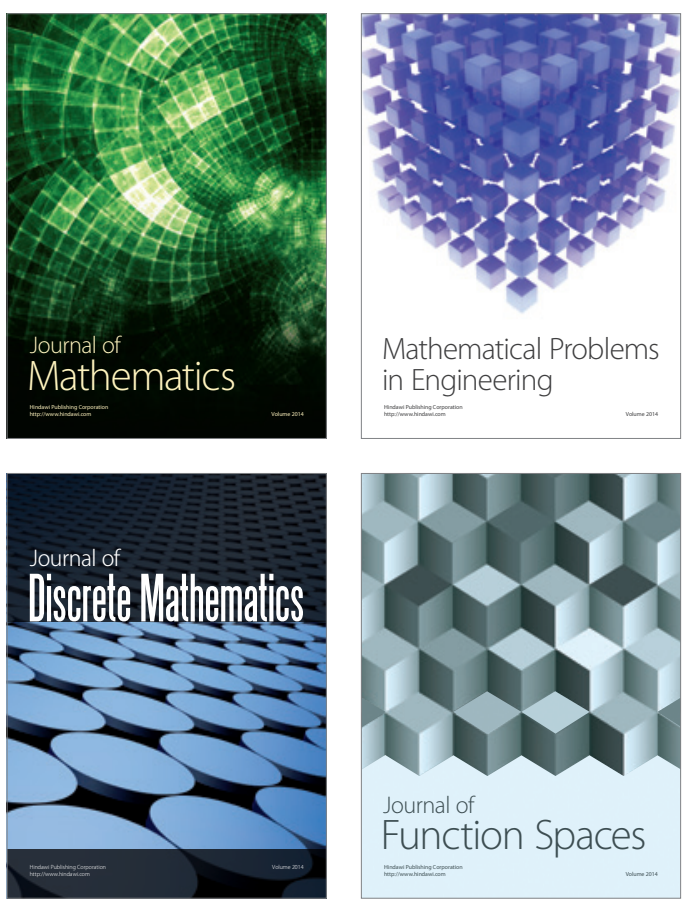

Mathematical Problems in Engineering
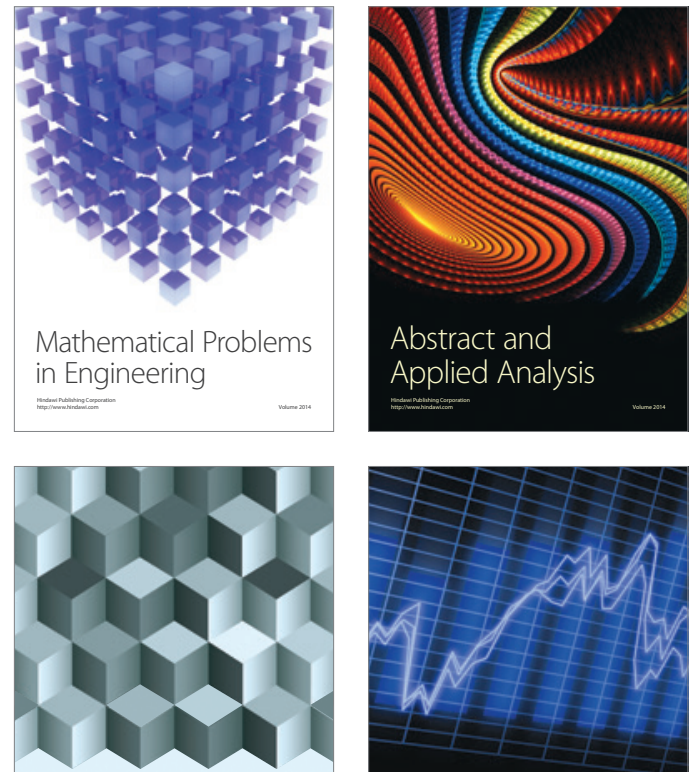

Journal of

Function Spaces

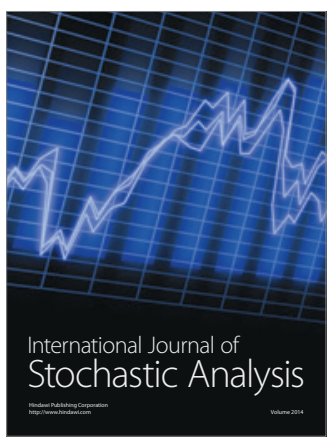

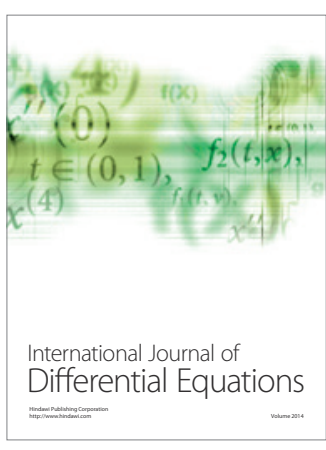
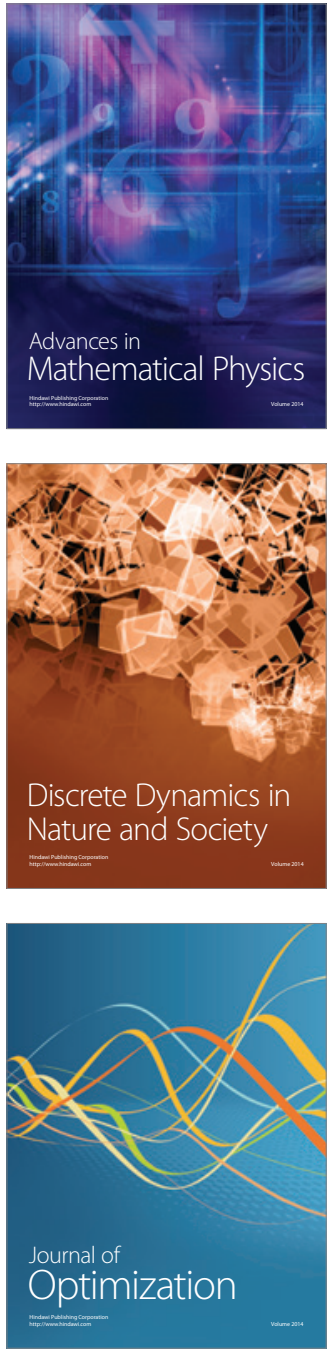